\title{
Grid-JQA: A QoS Guided Scheduling Algorithm for Grid Computing
}

\author{
Leyli Mohammad Khanli', Assistance Professor \\ and Saeed Kargar2, M.S. student \\ ${ }^{1}$ C.S. Dept., University of Tabriz \\ ${ }^{2}$ Islamic Azad University, Tabriz Branch
} Iran

\section{Introduction}

The development of grid computing technologies (Foster \& Kesselman, 2004) over the past several years has provided us a means of using and sharing heterogeneous resources over local/wide area networks, and geographically dispersed locations. This has resulted in the ability to form loosely coupled, high-performance computational environment comprising numerous scalable, fault tolerant, and platform-independent services across the entire Internet. The grid infrastructure provides a way to execute applications over autonomous, distributed and heterogeneous nodes by secure resource sharing among individuals and institutions. Typically, a user can submit jobs to a grid without necessarily knowing (or even caring) where it will be executed. It is the responsibility of the grid resource management system to distribute such jobs among a heterogeneous pool of servers, trying to optimize the resource usage and provide the best possible quality of service.

Thus, the resource matching problem is an important task in the Grid environment which involves assigning resources to tasks in order to satisfy task requirements and resource policies. This contribution presents algorithms, methods, and software for a Grid resource manager, responsible for resource brokering and scheduling in Grids. The broker selects computing resources based on actual job requirements and a number of criteria identifying the available resources, with the aim to minimize the turnaround time for the individual application.

In this chapter, we proposed Grid-JQA (Khanli \& Analoui, 2006a,b) that explains in next sections. The Grid Java based Quality of service management by Active database (Grid-JQA) is a framework that aims to address the above mentioned requirements. Grid-JQA provides management for quality of service on different types of resources, including networks, CPUs, and disks. It also encourages Grid customers to specify their quality of service needs based on their actual requirements. The main goal of this system is to provide seamless access to users for submitting jobs to a pool of heterogeneous resources, and at the same time, dynamically scheduling in multi policy mode and monitoring the resource requirements for execution of applications. We proposed an optimal solution but not practical for comparing with other practical solution.

Also, we propose an aggregation formula for the QoS parameters. The formula is a unit less combination of the parameters together with weighting factors. It is shown that the formula 
needs to put into a threshold consideration. A discussion on the threshold and its level is also provided. In this chapter we introduce an optimum but not practical solution for matching. The optimum method is considered for comparing other practical solutions. Main features of the resource manager include resource selection based on active database rules (Khanli \& Analoui, 2008) and environmental conditions and a basic adaptation facility. The chapter is finalized by the results obtained from simulation and a comparison study.

The reminder of this chapter is organized as follows: In section 2 we present an overview of related work, in section 3 we introduce three methods for matching in the grid environment. We discuss a proof-of-concept implementation of a prototype and the preliminary performance results by simulation in Section 4, whereas Section 5 concludes with a summary of our work.

\section{A brief history of the resource management in grid}

Due to the grid computing is a dynamic and uncertainly environment, the grid system needs a resource management mechanism which provides a set of available appropriate resources for requesters. There are many resource management approaches that proposed in different grid projects and researches, but a few of them guarantee QoS. This section provides a brief description of these systems and compares some of them with our project.

Foster et al. (Foster et al. 1999) propose a framework for QoS in Grid computing, called the Globus Architecture for Reservation and Allocation (GARA), which enables programmers and users to specify and manage end-to end QoS for Grid-based applications. It also provides a uniform mechanism for making QoS reservations for different types of Grid resources, such as processors, networks and storage devices. The main drawback of GARA is its inability to support subtask management, which is one of Grid's main goals. There are two more drawbacks in GARA: the topology of domain should be known in advance and also the resources can not publish themselves. Grid-JQA is going to address the subtask management while the topology information and resource publishing are handled management, formulating the matching problem as an object placement problem dynamically (Khanli \& Analoui, 2006a,b).

Y. Han et al. (Han \& Youn, 2009) proposed a new architecture that called Resource-Aware Policy Administrator (RAPA) with Service Level Agreement (SLA) to find appropriate resources. SLA operates as a contract agreed between users and resource providers in order to achieve requested QoS (Han \& Youn, 2009; Document for Web Services Agreement Specification).

Chauhan \& Joshi (2010a) proposed a QoS Guided Weighted Mean Time Min-Min Max-Min selective heuristic for QoS based task scheduling. In fact this work adds QoS parameters to weighted Mean Time Min-Min Max-Min Selective (WMTS) heuristic (Chauhan \& Joshi, $2010 \mathrm{~b})$. In this method, first the meta tasks divided into high and low QoS groups, that the high QoS group contains the tasks with high requirement QoS and the other one includes the tasks with low QoS requirements. In mentioned work after mapping all tasks with high Qos requirements, the tasks with low QoS group are mapped (Chauhan \& Joshi, 2010a).

Selvi Somasundaram et al. (2010) proposed a metascheduling structure that called CARE Resource Broker (CRB). For resource management they are proposed Virtual Resource Management Protocol (VRMP). In this method scheduler is responsible for choose a suitable resource between available matching resources, and Virtual resource manager is responsible for formation of virtual cluster and virtual machine (Somasundaram et al., 2010). 
Darby III \& Tzeng (2010) proposed a Checkpointing arrangement in Mobile Grid Computing that provide QoS through Mobile hosts. In other words current work focus on the Mobile hosts Checkpointing arrangement method that QoS is a user's application responsibility.

Rodero et al. (2010) proposed Grid broker selection strategies to resolve the broker selection problem in multiple grid scenarios (Rodero et al., 2010). They also proposed two different resource aggregation algorithms (SIMPLE and CATEGORIZED aggregation algorithms) that used for broker selection.

Javelin (Neary et al., 2000) is a Java based infrastructure for internet-wide parallel computing. The three key components of Javelin system are the clients or applications, hosts, and brokers. A client is a process seeking computing resources, a host is a process offering computing resources, and a broker is a process that coordinates the allocation of computing resources. The Javelin system can be considered a computational Grid for highthroughput computing. It has a hierarchical machine organization where each broker manages a tree of hosts. Resources are simple fixed objects with a tree namespace organization. The resources are simply the hosts that are attached to a broker. Any host that wants to be part of Javelin contacts JavelinBNS system, a Javelin information backbone that maintains the list of available brokers. The host then communicates with brokers, chooses a suitable broker, and then becomes part of the broker-managed resources. Thus the information store is a network directory implemented by JavelinBNS. Hosts and brokers update each other as a result of scheduling work. Thus, Javelin uses demand resource dissemination. The broker manages the host-tree or resource information through a heaplike data structure. Resource discovery uses the decentralized query based approach since queries are handled by the distributed set of brokers. In Javelin the burden of the subtask management and monitoring is layered on the client side. The client should also look for a broker that matches its requirements. Thus the clients are thick. Grid-JQA tries to keep the clients as thin as possible and refers the subtask management and monitoring, and the matching to the broker of the system (Khanli \& Analoui, 2006a,b).

Legion (Chapin et al., 1999) takes an object-oriented approach to resource (Chapin et al., 1999). The identification of a candidate resource is performed by an object mapper, whose recommendation is then implemented by a different object. The Legion system defines a notation (Chapin et al., 1999) that is similar to classAds, although it uses an object oriented type system with inheritance to define resources, as contrasted with the simple attributeoriented Boolean logic of classAds. Legion supports autonomy with a jurisdiction magistrate (JM), which may reject requests if the offered requests do not match the policy of the site being managed by the JM. While the JM gives a resource veto power, there is no way for a resource to describe those requests that it would rather serve (Khanli \& Analoui, 2006a,b).

UDDI (Uddi.com) and eSpeak (Hewlett Packard) are two specifications being defined to enable automation of business-to-business interactions. Both systems use XML as a specification language to describe services, and define a rich framework for service discovery. Like most other systems, neither UDDI nor eSpeak exports a symmetric interface to servers and customers. Furthermore, since the emphasis in these frameworks is on service discovery and not resource allocation, the matchmaker provides a list of candidate servers to the customer, who then chooses one or more servers based on subjective criteria.

Recently, to solving the grid resource management problem, many of technologies proposed that based on matchmaking or bidding (Fig. 1). In the matchmaking (Khanli \& Analoui, 2008; Litzkow et al., 1988; Liu et al., 2002; Ludwig \& Reyhani, 2006; Raman et al., 1998; 
Raman et al., 2003; Tangmunarunkit et al., 2003; Yu et al., 2005), there are a resource matchmaker that responsible for recording resource status. When a resource matchmaker gives job requests, executes matching algorithms to find a set of appropriate resources for request.
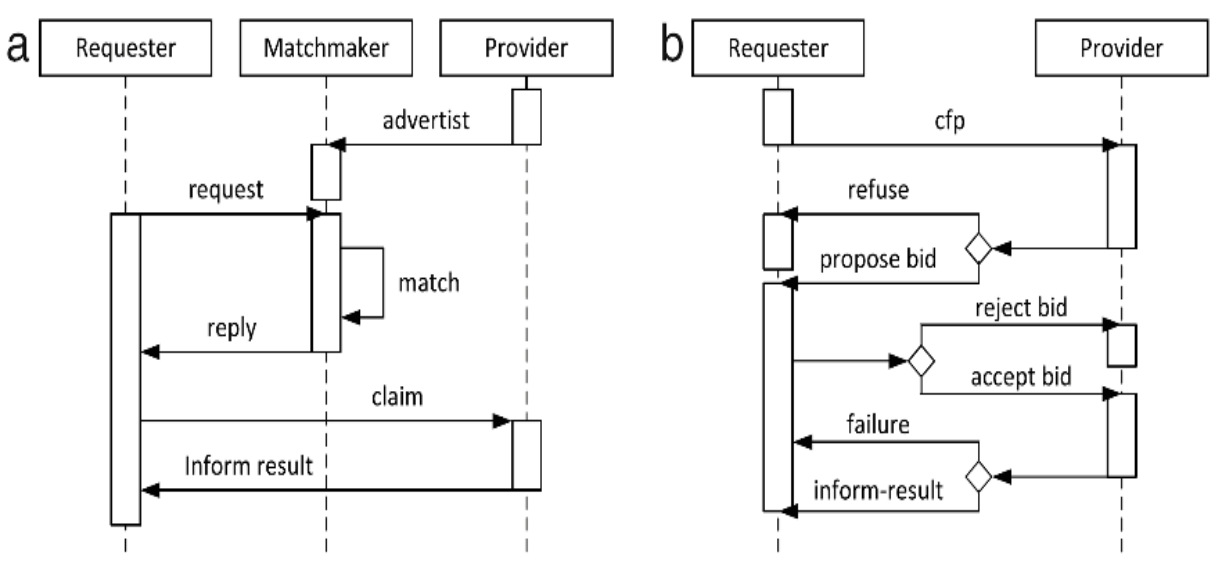

Fig. 1. (a) The abstract process of the matchmaking model. (b) The abstract process of the bidding model (Wang et al., 2010).

Another model for resource management is bidding-based model (Bubendorfer, 2006; Buyya et al., 1980; Das \& Grosu, 2005; Goswami \& Gupta, 2008; Kakarontzas \& Savvas, 2006; Kolano, 2004; Leal et al., 2009; Smith, 1980; Wang et al., 2010; Xiao et al., 2008). In this model there is no centralized matchmaker/broker. However, the bidding model has some advantages over the matchmaking one, but in this model the critical problem for resource selection is that there is no universal information to find suitable match, and requests only have deficient information (Wang et al., 2010).

The Condor (Raman et al., 2003) equivalent of an information system such as UDDI is a matchmaker that maintains a pool of ClassAds representing candidate resources and then matches each incoming request ClassAd with all candidates, returning a highest-ranked matching candidate. Redline (Liu \& Foster, 2004) formulates the matching problem as a constraint satisfaction problem. These latter systems allow expression of resource groups, but they do not offer a concise method to express network topologies. Set-matching extends the ClassAds language to provide a multilateral matchmaking mechanism where the number of resources is not known a priory (Liu et al., 2002). However, the set matching mechanism is not capable of marshaling a heterogeneous mix of resources.

Our work is similar to Condor. That is the resources advertise themselves to the broker and the users send their requests to the broker. Then the broker matches the resources and the tasks by using some policies. The differences between our work and Condor are after this time. Because, after matching the broker does not interfere in Condor. And the user works with resources directly. But in our work, the broker manages the activities till the results return to the user. The disadvantage of managing broker till the end is that the broker becomes too busy. For correcting this disadvantage, we use hierarchical broker, in a way that when the broker becomes too busy, one new broker is introduced in the low level and 
busy broker can send new application for execution to lower level broker. So the problem is solved by hierarchical broker.

\section{Model description}

Looking on the matching problem, we bring about the following solutions:

First, resources introduce themselves to all clients and each client decides where to send its request, thus matching is accomplished by the client.

Second, the clients send their request to all resources and each resource decides which request to reply, therefore matching is done in the resources.

Third, the resources introduce themselves to a broker and each client sends its request to the broker, then the broker decides how to match the requests with the resources. Here there are two possible methods, (a) after the broker finds the best matched resource; the resource and the client negotiate with each other directly. (b) The broker interferes in the process until end of job, in order to guarantee the quality of service and perform subtask management.

In this work, we implement the third mentioned solution (b). The advantages of the broker management till end are:

1. For guarantying the QoS parameters, the broker should monitor till the results return to the user.

2. May be all requested resources are not free, so application can not start execution, but in our work as soon as one resource is free, the execution of user's task can be started. Gradually, the remained tasks can be executed when the other resources are released.

3. Adaptation is possible only in this method that the broker monitors till the end, as duplicate execution of strong tasks which are being executed in weak resources.

4. By using the broker, the user can be thin client that is the users can execute their application by PDA or Mobile handset considering the user want the power of processing like supercomputer. Because the broker manages for matching, monitoring, sending task, receiving results, and managing failed tasks, the user only sends the request and waits until it receives the results.

5. Managing failed tasks is simpler than the other methods. Because the broker can assign more priority to failed tasks and matches them as soon as possible.

6. Fault tolerance is possible. The broker can execute the tasks with more priority in duplicate mode.

\section{Matching}

Efficient resource selection in every resource management approach is one of the major challenges. There is one problem about matching in grid environment. Following model explains the problem:

1. The problem input

1.1. A set of resources with their capabilities

1.2. A set of tasks with their requirements

2. The problem output: Matching the best resource for each task

3. The problem purpose: Minimizing turnaround time

There are two managing models:

- Local

- Global 
In Local model, the manager finds the best resource for each task locally regardless of other tasks. Whereas in Global model, the manager finds the proper resource for each task in relation with other tasks and the strong resource is assigned to strong task (The task is strong / weak if its requirement is high / low). Whereas in local model the manager may assign the strongest resource to first task that may be the weakest one. Therefore for the next task which may happen to be a strong task, the strong resource doesn't exist anymore. So it is better to apply the matching management in a global model.

We propose three methods for matching. They are compared using simulation in the next section. Three methods are:

1. Optimum

2. Grid-JQA

3. Dup Grid-JQA

That we can consider:

- $\mathrm{n}$ : the task number

- $\mathrm{m}$ : the resource number

- $\quad \mathrm{k}$ : the number of QoS parameters

As it was explained, the resources should introduce themselves to the broker. Each introduction includes resource specifications as CPU, Memory, etc. Using schedulers, such as (Xen Scheduler Howto, 2005), a resource can assign a percentage of CPU to a given machine effectively regulating the CPU usage of the group of processes encapsulated in it. And also it includes some parameters such as the bandwidth and the delay were calculated or estimated by the broker. Finally the broker inserts the record of QoS parameters for each resource into the database. We define the vector $q^{\text {res }}$ which gives the capabilities of a resource as follows.

$$
q^{r e s}=\left\langle q_{1}^{r e s}, q_{2}^{r e s}, \ldots, q_{k}^{r e s}\right\rangle
$$

Generally it can be said that the client sends its request accompanied by vector of QoS parameters and the weights for the parameters as it appears in equations 2 and 3.

$$
\begin{gathered}
q^{\text {task }}=\left\langle q_{1}^{\text {task }}, q_{2}^{\text {task }}, \ldots, q_{k}^{\text {task }}\right\rangle \\
\left.\mathrm{W}=<\mathrm{w}_{1}, \mathrm{w}_{2}, \ldots, \mathrm{w}_{\mathrm{k}}\right\rangle \quad 0 \leq \mathrm{w}_{\mathrm{i}} \leq 1 \quad \sum_{i=1}^{k} \mathrm{w}_{\mathrm{i}}=1
\end{gathered}
$$

Each weight is used to show the importance of each parameter. For example, if CPU is important for one task, the client will set 1 for the CPU weight and zero for the others.

Note that, $\mathrm{q}_{\mathrm{i}}^{\text {res }}$ and $\mathrm{q}_{\mathrm{i}}^{\text {task }}$ have the same unit, and the broker compares $\mathrm{q}_{\mathrm{i}}$ res with $\mathrm{q}_{\mathrm{i}}^{\text {task }}$ for each $\mathrm{i}$ from 1 to $\mathrm{k}$. If the resource provides the requirements needed for the task, it can be chosen as the best matched resource. We introduce satisfy operator $\backsim R_{i} \bowtie T_{j}$ means that the resource Ri can satisfy the task $\mathrm{Tj}$ and guarantees QoS parameters.

$$
\mathrm{R}_{\mathrm{i}} \text { Ф } \mathrm{T}_{\mathrm{j}}=\left(\left(\sum_{l=1}^{k} \frac{q_{l}^{\operatorname{Res}_{i}}}{q_{l}^{\text {task }} k_{j}} \times w_{l}\right) \geq 1\right) \quad(\mathrm{k}=\text { the number of QoS parameters })
$$

The solution proposed here is that, we normalize the resource specification by the client requirement ( $\mathrm{q}_{\mathrm{i}}^{\text {res }} / \mathrm{q}_{\mathrm{i}}^{\text {task }}$ ) and therefore the summation will be possible whereas the units of 
each parameter are different such as byte, bps, Mflops. When the resource capability exceeds the task demand, ( $\mathrm{q}_{\mathrm{i}}^{\mathrm{res}} / \mathrm{q}_{\mathrm{i}}^{\text {task}}$ ) is more than one.

Also, the client introduces a weight for each parameter to show the importance of the parameter. The weights range from 0 to 1 and the sum of all the weights is equal to one. We multiply the weight into ( $\mathrm{q}_{\mathrm{i}}^{\text {res }} / \mathrm{q}_{\mathrm{i}}^{\text {task}}$ ) as mentioned in (4). Finally the best match resource will be the one that can provide the maximum of summation in (4).

Note that matching is done by aggregating QoS parameters which simplifies the matching method. You may say that using aggregated QoS parameters, some requirements may be neglected. This argument can be addressed by the introduction of the proper weights. If user assigns a higher weight for a QoS parameter, the requirement surely will be met.

The proposed aggregation in (4) has one more advantage, whereas the overheads and matching time is concerned. It is obvious that the matching time for aggregated QoS is a fraction of time when we do not aggregate the k parameters.

The three methods for matching are proposed by using summation in (4).

\subsection{Optimum}

Assigning $\mathrm{m}$ resources to $\mathrm{n}$ tasks is the NP complete problem. The Optimum method has time complexity $\mathrm{O}\left(n^{3}\right)$ to find the best matched resource for each task globally. Practically the Optimum method cannot be used because it has high overhead. We take into consideration the Optimum solution just for the sake of the comparison.

The problem is assigning $\mathrm{m}$ resources to $\mathrm{n}$ tasks with $\mathrm{k}$ QoS parameters. There are three matrices, one is $T_{n^{*} k}$ matrix given by (5) for task requirements, another is $W_{n^{*} k}$ matrix given by (6) for weight of requirements, and the other is $R_{\mathrm{k}^{*} \mathrm{~m}}$ matrix given by (7) for resource capabilities. These matrices are shown in below.

$$
\begin{aligned}
& T_{n^{*} k}=\left[\begin{array}{cccc}
q_{1}^{\text {task }_{1}} & q_{2}^{\text {task }_{1}} & \cdots & q_{k}^{\text {task }_{1}} \\
q_{1}^{\text {task }_{2}} & q_{2}^{\text {task }_{2}} & \cdots & q_{k}^{\text {task }_{2}} \\
\vdots & \vdots & \ddots & \vdots \\
q_{1}^{\text {task }_{n}} & q_{2}^{\text {task }_{n}} & \cdots & q_{k}^{\text {task }_{n}}
\end{array}\right] \\
& W_{n^{*} k}=\left[\begin{array}{cccc}
w_{1}^{\text {task }_{1}} & w_{2}^{\text {task }_{1}} & \cdots & w_{k}^{\text {task }_{1}} \\
w_{1}^{\text {task }} & w_{2}^{\text {task }} & \cdots & w_{k}^{\text {task }} \\
\vdots & \vdots & \ddots & \vdots \\
w_{1}^{\text {task }} & w_{2}^{\text {task }} & \cdots & w_{k}^{\text {task }}
\end{array}\right] \\
& R_{k^{*} m}=\left[\begin{array}{cccc}
q_{1}^{r e s_{1}} & q_{1}^{r e e_{2}} & \cdots & q_{1}^{r e s_{m}} \\
q_{2}^{r e s_{1}} & q_{2}^{r e s_{2}} & \cdots & q_{2}^{r e s_{m}} \\
\vdots & \vdots & \ddots & \vdots \\
q_{k}^{r e s_{1}} & q_{k}^{r e e_{2}} & \cdots & q_{k}^{r e s_{m}}
\end{array}\right]
\end{aligned}
$$

We define $\mathrm{WdT}_{\mathrm{n}^{* k} \mathrm{k}}$ matrix as below. 


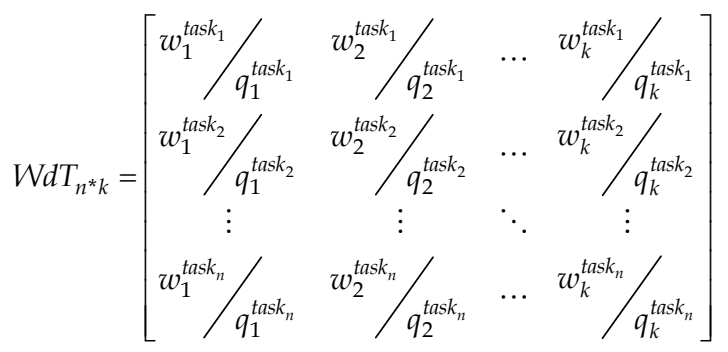

So, equation (4) is based on multiplying $\mathrm{WdT}_{n^{*} \mathrm{k}}$ matrix to $\mathrm{R}_{\mathrm{k}^{*} \mathrm{~m}}$ matrix and the result is $\mathrm{V}_{\mathrm{n}^{*} \mathrm{~m}}$ matrix given by (9) and (10).

$$
\begin{aligned}
& V_{n^{*} m}=W d T_{n^{*} k}{ }^{*} R_{k^{*} m}
\end{aligned}
$$

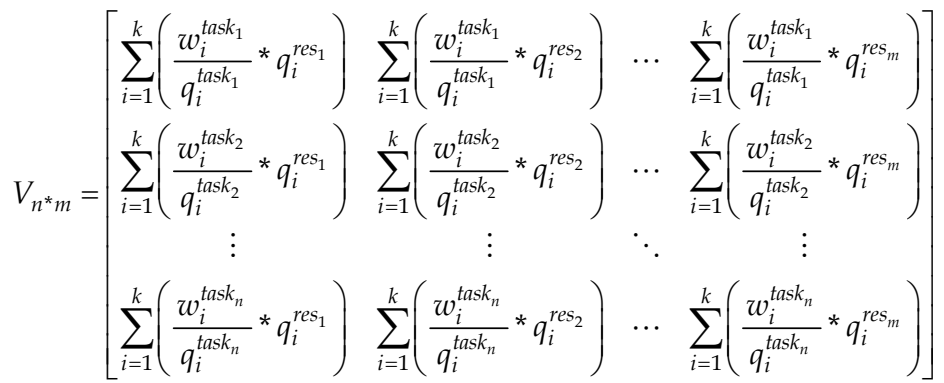

$V_{i, j}$ shows the value of (4) for assigning resource $j$ to task $i$. If $V_{i, j}=1$, the resource $j$ exactly will provide the task $i$ requirements. If $V_{i, j}<1$, the resource $j$ will be weaker than task $i$ requirements. If $V_{i, j}>1$, the resource $j$ will be stronger than task $i$ requirements.

We define MAX unary operator that it gets one $n * m$ matrix and produces $n * 3$ matrix. In the following, the operator semantic is explained.

$$
\begin{aligned}
& B_{n^{*} 3}=M A X A_{n^{*} m}
\end{aligned}
$$

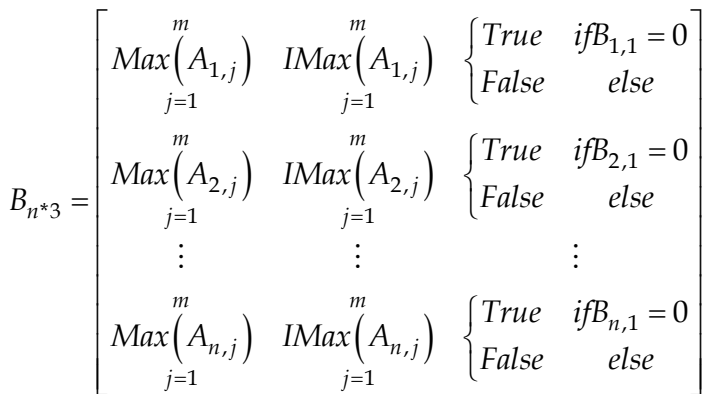

Max returns the maximum value for each row of operand and IMax returns the index of maximum value in each row of operand. Third column of matrix $B$ shows that if the first column of matrix B is zero, it will be True (i.e. this row's task is tested for matching) otherwise it will be False (i.e. this row's task is not matched yet). So we define matrix $M_{n \star 3}$ as below: 


$$
M_{n^{* 3}}=\operatorname{MAX} V_{n^{*} m}
$$

Fig. 2 shows the Optimum algorithm that matches the best resource for each task globally. The algorithm finds the values of the assignment of each resource to $n$ tasks at first. The purpose is assigning the maximum value resource for each task. Next, the algorithm finds the maximum value at each row which belongs to a task. It is possible that the resource with maximum value in one row may be also is maximum in other rows. Because of that, the algorithm chooses the row (task) which has minimum value (this task has maximum requirements). If the number of matching becomes equal to number of tasks (n) (that is the all of third column of matrix $\mathrm{M}$ is True), then the algorithm will finish.

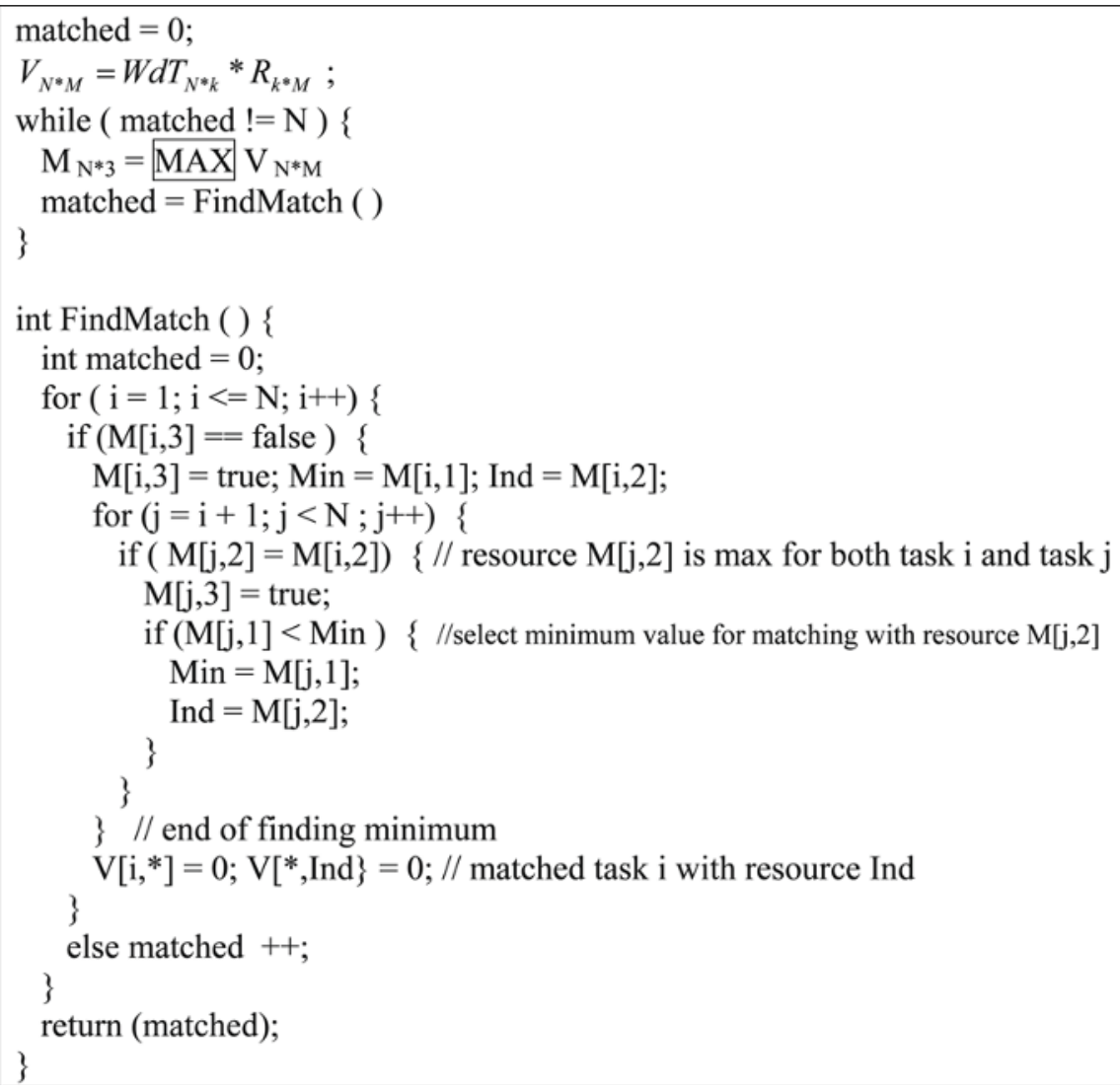

Fig. 2. Optimum Algorithm

\subsection{Grid-JQA}

Grid-JQA has time complexity $\mathrm{O}(n)$ instead of $\mathrm{O}\left(n^{3}\right)$ for Optimum method and (as shown in next section by simulation), the difference between Grid-JQA and Optimum is acceptable. Grid-JQA uses threshold in (4) instead of finding maximum and minimum for each assignment (Fig. 2). 


$$
\operatorname{Ri} \text { ๗ } \mathrm{Tj}=\left(\left(\sum_{l=1}^{k} \frac{q_{l}^{\operatorname{Res}_{i}}}{q_{l}^{\text {task }}} \times w_{l}\right) \geq \text { Threshold }\right)(\mathrm{k}=\text { the number of QoS parameters })
$$

The proposed solution is that instead of using maximum, we use a threshold (Threshold in (14)). If summation is more than the Threshold, the broker will choose it as the best matched resource.

The broker starts calculating (14) for record $R_{1}$. It keeps calculation till finds $R_{i}$ that satisfies the task. So the broker assigns $R_{i}$ to the task. For next task to be matched, the broker starts from $R_{i+1}$ in a round robin fashion, so that all resources can be covered.

Now, the problem is "what can be the amount of the threshold? ". For solving this problem, tacking into consideration that the active database is used, we introduce a rule in active database for calculating threshold in relation to environmental changes.

Suppose $\mathrm{q}_{\mathrm{k}}^{\mathrm{R}}$ equals $\mathrm{q}_{\mathrm{k}}^{\mathrm{T}}$ for all $\mathrm{k}$ from 1 to $\mathrm{n}$ to $\mathrm{n}$ (i.e. the requirements of task equal the capabilities of resource), then:

$$
\sum_{k=1}^{n} \frac{q_{k}^{R i}}{q_{k}^{T j}} \times w_{k}=\sum_{k=1}^{n} 1 \times w_{k}=1
$$

So the minimum of threshold is 1 .

If $\mathrm{q}_{\mathrm{k}}^{\mathrm{R}}$ equals $\mathrm{P}^{*}\left(\mathrm{q}_{\mathrm{k}}^{\mathrm{T}}\right)$ for all $\mathrm{k}$ from 1 to $\mathrm{n}$, then:

$$
\sum_{k=1}^{n} \frac{q_{k}^{R i}}{q_{k}^{T j}} \times w_{k}=\sum_{k=1}^{n} P \times w_{k}=P
$$

Therefore if we assign $\mathrm{P}$ to the threshold, it means that the selected resource should provide $P$ times capability more than the requirements by the task.

Therefore when there are a lot of strong and free resources it is better that the broker assigns the strong resource to weak task. So the Threshold should be greater than one and the broker should select a resource precisely. In this method Grid-JQA acts like an Optimum method. But when there are a few strong resources it is better that to set the threshold equal one. So the broker should select a resource as soon as it finds a free resource and the broker does not wait for strong resources.

The value of threshold is changed by a rule of active database related to the number of times that equation 14 returns true or false. In this way if the number of true is more than false, the rule should be increased threshold for finding strong resource and otherwise should be decreased threshold for assigning resource to task as soon as possible.

Next section shows that Grid-JQA is similar to Optimum using simulation. But Grid-JQA has time complexity $\mathrm{O}(\mathrm{n})$ instead of time complexity $\mathrm{O}\left(\mathrm{n}^{3}\right)$ for Optimum.

\subsection{Dup Grid-JQA}

After assigning $\mathrm{m}$ resources to $\mathrm{n}$ tasks, definitely some tasks are executed in weak resources which results is increasing the turnaround time of entire application. Dup Grid-JQA assigns free strong resources to tasks executed in weak resources in duplicate mode. Reference (Kondo, 2005) shows that for the application with 100 tasks, $90 \%$ of the tasks are completed in about 39 minutes, but the application does not finish until 79 minutes have passed, which is almost identical to the turnaround time for a much larger application with 400 tasks. 
There are two main causes of this plateau. The first cause is task failures that occur near the completion of the application. When a task fails, it must be restarted from scratch, and when this occurs near the end of the application execution, it will delay the application completion. The second cause is tasks assigned to slow resources. Once a task is assigned to a slow host, a FCFS scheduler without task preemption or replication capabilities will be forced to wait until the slow resource produces the result.

Dup Grid-JQA is a solution for the second cause. As when the $60 \%$ of execution time of application completes Dup Grid-JQA schedules remaining tasks in duplicate mode. In duplicate mode, the broker will send that results which is obtained first. Next section shows that duplicate method decreases the turnaround time significantly.

As the number of tasks gets large in comparison with the number of resources in the platform, the plateau becomes less significant, thus justifies the use of a FCFS strategy. However, for applications with a relatively small number of tasks, resource selection could improve the performance of application significantly.

\section{Simulation}

There are three subsections: 1) Application Definition 2) Resource definition 3) Matching methods and comparisons.

\subsection{Application definition}

We use XML as input language. Fig. 3 shows one example of application definition in simulation.

The random function is used to provide random number between Min and Max for each field in simulation so that the environment gets more similar to grid environment that rapidly changes.

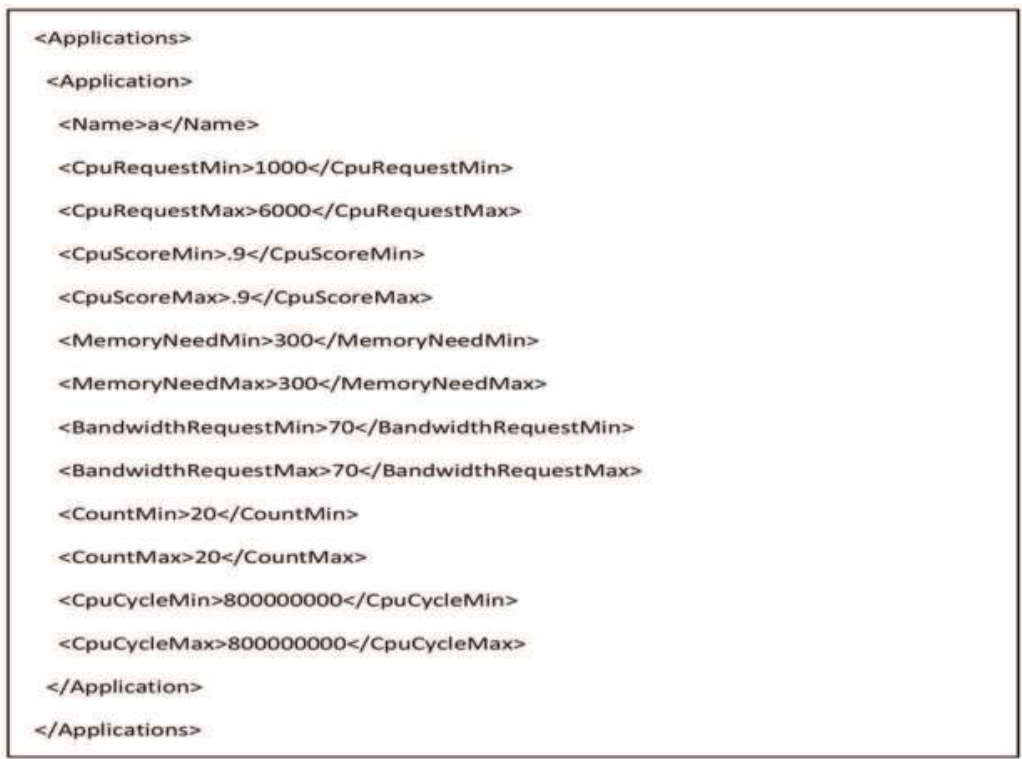

Fig. 3. Application Definition 


\subsection{Resource definition}

Fig. 4 shows one example of resource definition by XML language. The requirement of memory must be completely satisfied by the resource, but the assignment of the memory more than requirements can not improve performance. Thus the requirement of memory is not used in aggregated QoS parameters in equation 14. Therefore the weight of bandwidth is the weight of CPU subtracted from one. The CPU power and bandwidth have a main role in improving turnaround time and if the broker assigns stronger CPU or more bandwidth, this assignment will cause the reduction of turnaround time. The application definition consists of the following fields:

1. Min/Max of CPU power requirement in cycle/sec (CpuRequestMin/Max)

2. Min/Max of CPU requirement's weight (CpuScoreMin/Max)

3. Min/Max of memory requirement (MemoryNeedMin/Max)

4. Min/Max of bandwidth requirement (BandwidthRequestMin/Max)

5. Min/Max of the number of tasks in application (CountMin/Max)

6. Min/Max of size of tasks in cycle/sec (CpuCycleMin/Max)

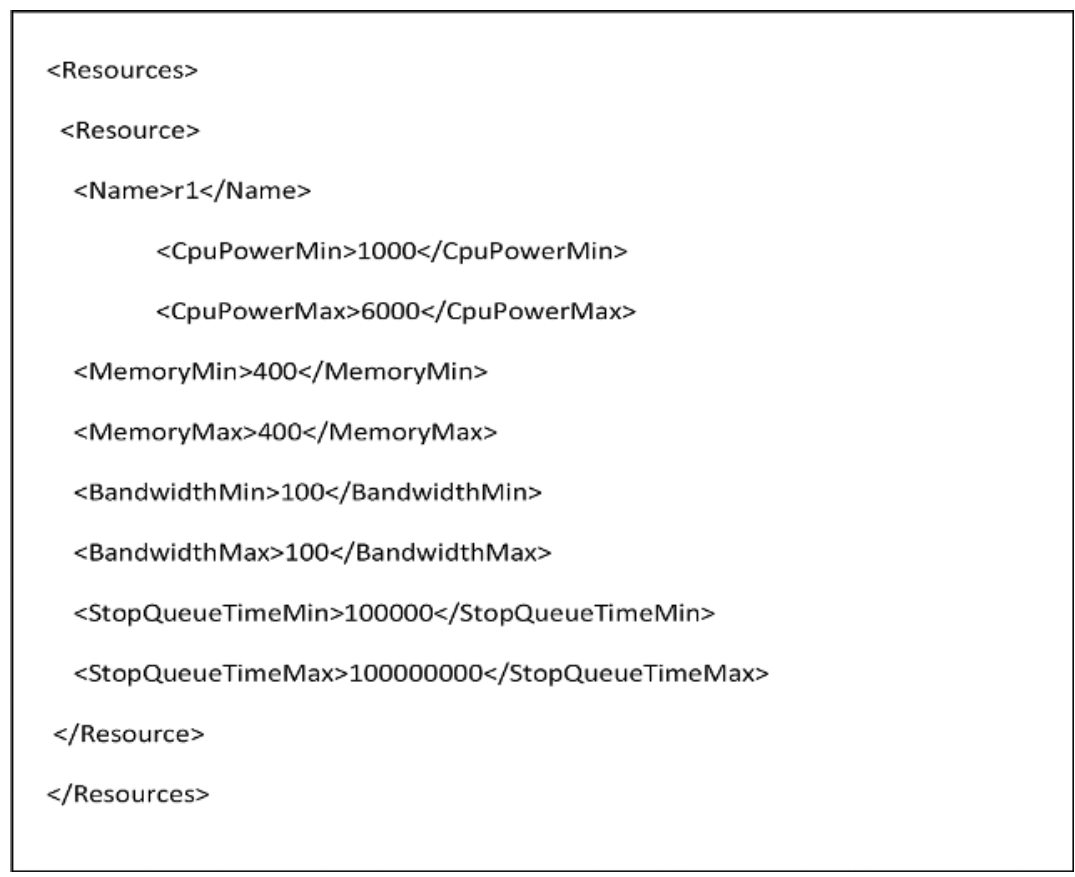

Fig. 4. Resource Definition

The resource definition consists of the following fields:

1. Min/Max of CPU cycle capabilities in cycle/sec (CpuPowerMin/Max)

2. Min/Max of memory in KB (MemoryMin/Max)

3. Min/Max of available bandwidth from the resource to the broker (BandwidthMin/Max)

4. Min/Max of availability duration for the resource (StopQueueTimeMin/Max)

As the application definition, the random function generates the random number between Min and Max for each above fields. 


\subsection{Matching methods and comparisons}

In simulation we use following five methods for matching:

1. The General method that matches resources with tasks in FIFO mode (first task to first free resource) regardless of QoS parameters.

2. The Optimum method, as described in section 4.1, selects the best resources for tasks but it has time complexity $\mathrm{O}\left(\mathrm{n}^{3}\right)$.

3. The Grid-JQA, our proposed solution, does matching almost like Optimum method but it has much less time complexity $(\mathrm{O}(\mathrm{n}))$ than Optimum matching.

4. Dup Grid-JQA, out proposed solution, that adds new feature to Grid-JQA. This feature is duplicate execution of delayed tasks (i.e. executed in weak resources).

5. The Wait method. In all other methods, if the broker does not find the proper resource, it will assign the best available resource to task. So the task will not wait for the proper resource. But in the Wait method tasks wait until the proper resource is found.

In this simulation, we assume that the CPU cycle is from 1000 to 6000, and all resources have same amount of memory and bandwidth. The number of tasks in application is 20 and the CPU weight is 0.9 . All tasks require same amount of memory and bandwidth.

We choose the CPU cycle requirement of application in range 1000 to 6000 which is similar to the range chosen for resource's CPU cycle power. We do simulation 6 times and each time we consider the amount of CPU cycle requirement 1000, 2000, 3000, 4000, 5000 and 6000. For each CPU cycle requirement, the simulation is repeated 100 times and finally we use the average turnaround time.

Fig. 5 shows the result of simulation for 6000 CPU cycle requirement with resource number from 10 (half of the task number) to 60 (three time more than the task number). After this, in all figures, horizontal axis indicates the number of the resources, and the vertical axis indicates the turnaround time in msec.

The simulation results show that:

1. Executing in weak resource in comparison with waiting to proper resource produces less turnaround time.

2. For strong requirement Grid-JQA is like Optimum method. Because, if Grid-JQA does not find the proper resource, it will assign the best resource as like Optimum.

3. General method does not change after 20 free resources, because it uses the 20 first free resources.

4. Dup Grid-JQA has minimum turnaround time when the number of free resource is from 10 (half of the task number) to 30 (1.5 times more than the task number). Because there are some tasks executed in weak resources (e.g. 1000-2000 CPU cycle/sec), they can find chance of executing in strong resources (e.g. 5000-6000 CPU cycle/sec). So the turnaround time is decreased.

5. When the number of free resources is less than the number of tasks, the Optimum method and Grid-JQA do not have better performance than the General method. They improve the results when the number of free resources gets more than the number of tasks. This situation is more likely because we suppose the environment has a lot of free resources (e.g. Internet).

6. Also, when the number of free resources is more than two times of the task number, the results are not improving.

Comparing below figures, we can find following results:

1. Most of the time, Grid-JQA has less turnaround time than Wait method (figures 5, 6, and 10). Only when the CPU cycle request is low (e.g. 2000 and 3000) and the number of free resource is from 10 ( half of the task number) to 30 (1.5 times ore than the task 


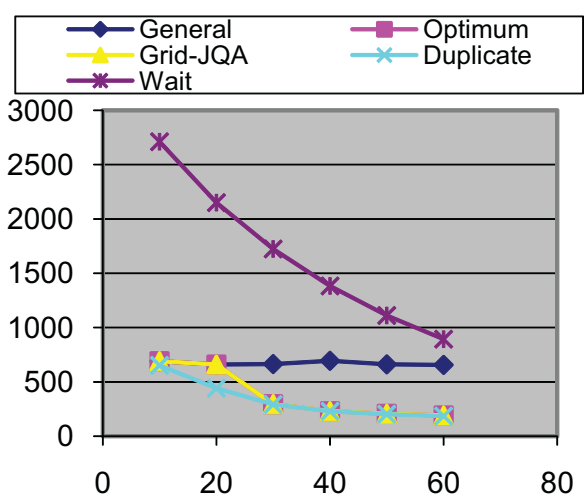

Fig. 5. 6000 CPU cycle request

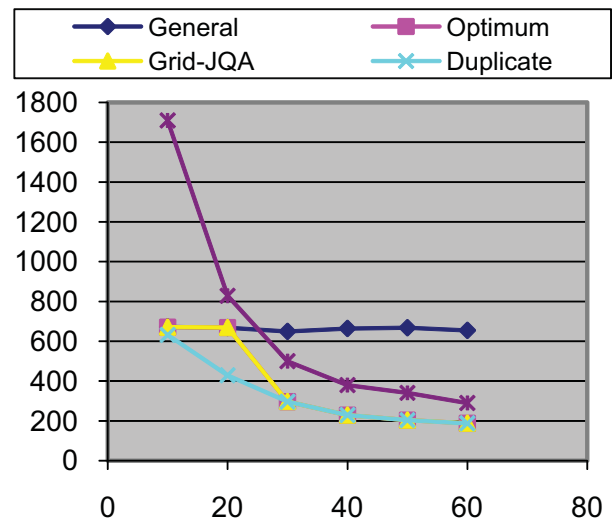

Fig. 6. $5000 \mathrm{CPU}$ cycle request

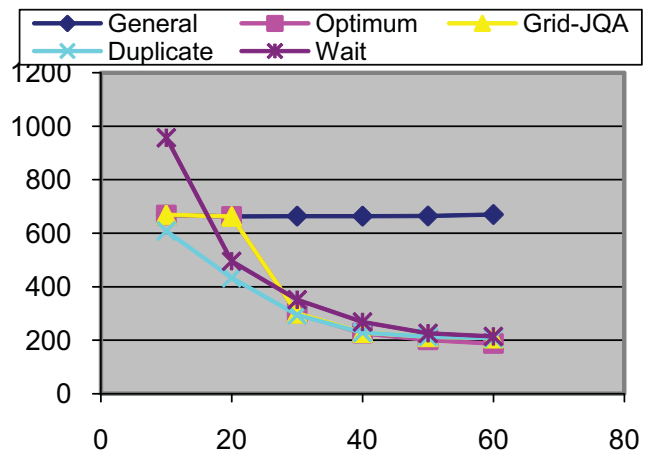

Fig. 7. 4000 CPU cycle request 


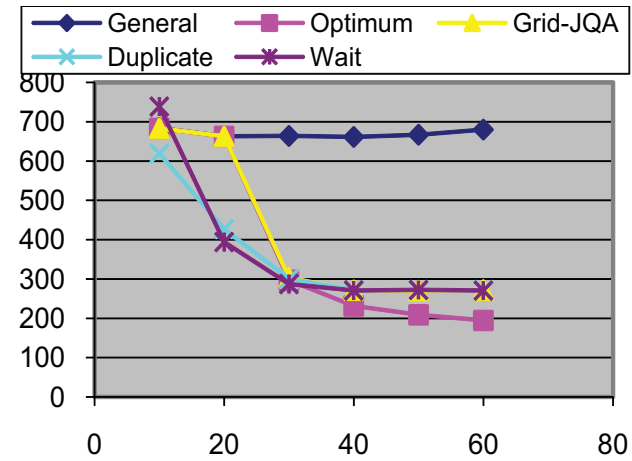

Fig. 8. $3000 \mathrm{CPU}$ cycle request
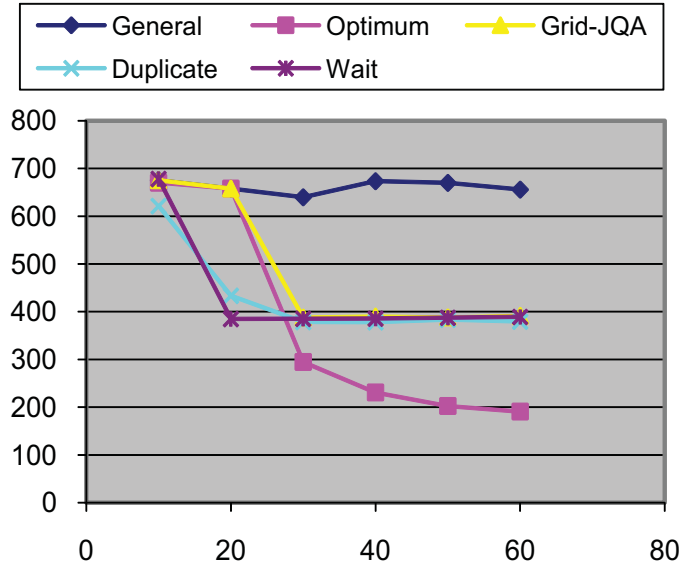

Fig. 9. 2000 CPU cycle request

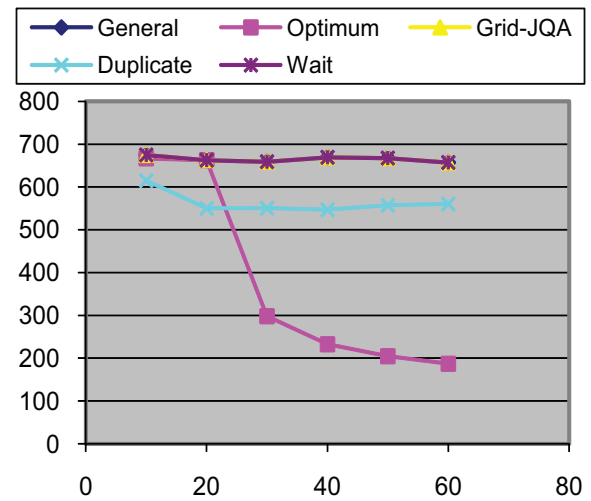

Fig. 10. 1000 CPU cycle request 
number), the Wait method has less turnaround time (Figures 8 and 9). So executing in weak resource is better than waiting for proper resource.

2. Comparing Grid-JQA and Optimum method shows that they are same for strong request (e.g. 4000-6000) in fig. 5, 6 and 7. The difference for 3000 CPU cycle request is ignorable. The difference between Grid-JQA and Optimum is for low requests (e.g. 1000-2000) that Optimum method has less turnaround time than Grid-JQA. But two points should be noted: First the user has low request so the execution time is longer and if the user wants less execution time, it should require more capabilities. Second, in this simulation the threshold is one, but as explained before, the threshold can be changed dynamically in related to environment changes so Grid-JQA can be similar to Optimum method.

For example if the CPU cycle request is 1000 and the threshold is 2, as shown in fig. 11 the difference between Grid-JQA and Optimum will be decreased. By increasing the threshold, the difference decreases. So with changing the threshold, Grid-JQA approaches to Optimum method.

3. Grid-JQA, our proposed solution, can provide the results close to the Optimum method and has the advantage of time complexity $\mathrm{O}(\mathrm{n})$ instead of $\mathrm{O}\left(\mathrm{n}^{3}\right)$.

4. Most of the time Dup Grid-JQA has less turnaround time in comparison with other methods. And also it has less cost in comparison with retrying and check pointing.

Finally, we do simulation 100 times with resources randomly between $(1000-6000)$ and application with requests randomly between $(1000-6000)$ with 20 tasks. Fig. 12 shows the results of this simulation.

Final results are:

1. Wait method has not better performance and starting execution in available resource is better than waiting for a suitable resource.

2. Adaptation (Dup Grid-JQA) has good results that is tasks executed in weak resources, can be executed in duplicate mode in strong resources. And the broker uses the results produced sooner.

So Dup Grid-JQA is suitable and reliable method for matching in grid environment.

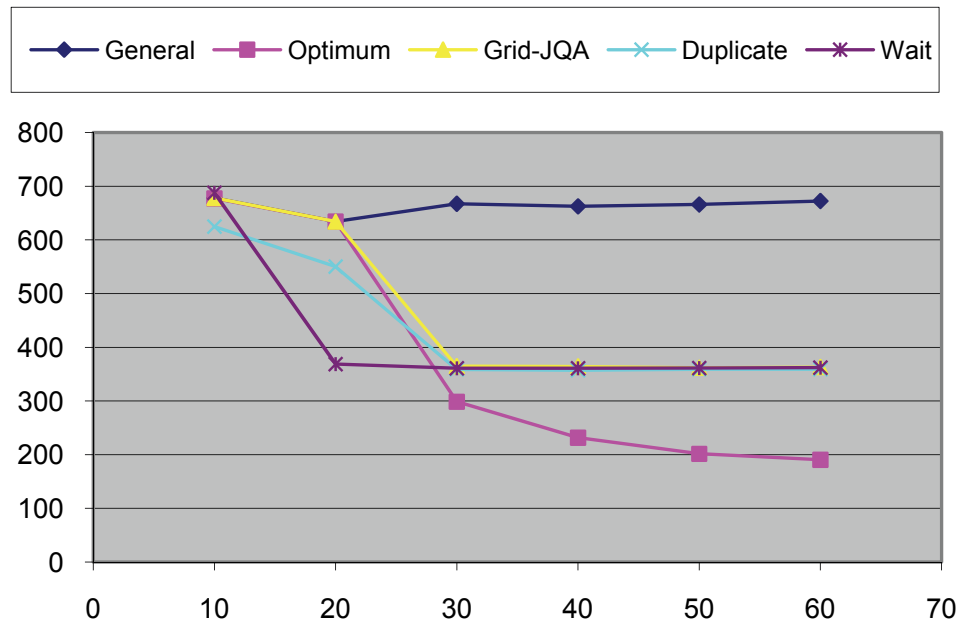

Fig. 11. 1000 cpu cycle request with threshold is 2 


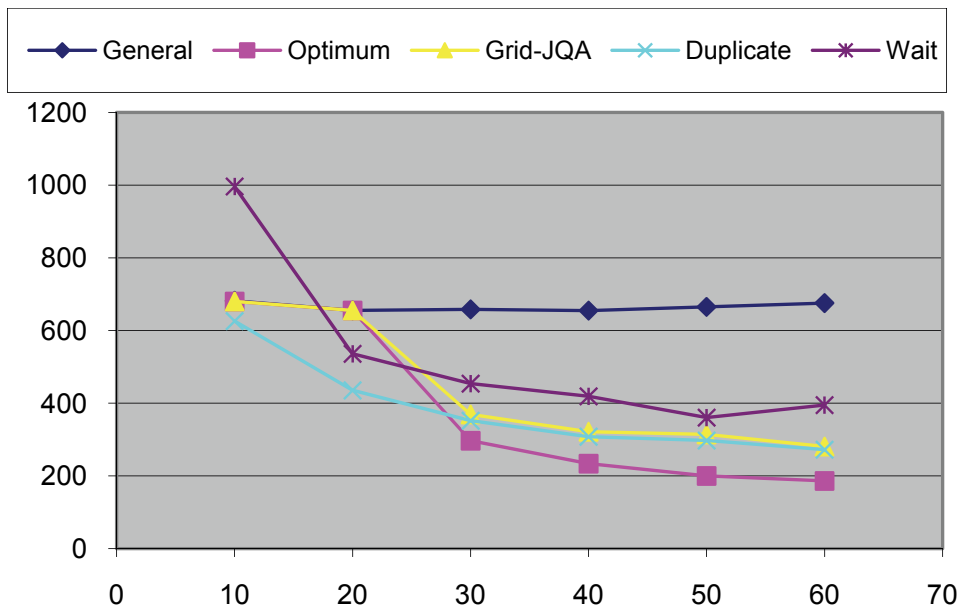

Fig. 12. Simulation for random request (1000-6000) and random capabilities (1000-6000)

\section{Conclusions}

This chapter discusses the matching methods in grid environment when QoS parameters are concerned. The Optimum method for matching $\mathrm{n}$ tasks with $\mathrm{m}$ resources is proposed by using matrices formalism. Grid-JQA and Dup Grid-JQA are proposed and compared with Optimum method.

Three heuristic approaches have been designed and compared via simulations to match tasks which take into account the QoS requested by the tasks, and at the same time, to minimize the tasks makespan as much as possible.

The Optimum method has time complexity $\mathrm{O}\left(\mathrm{n}^{3}\right)$ that is decreased to $\mathrm{O}(\mathrm{n})$ by using GridJQA with ignorable differences. The Dup Grid-JQA resource selection selects resources based on threshold scheduling and fault tolerance by duplicating the execution of failed tasks or delayed tasks. The simulation shows that Dup Grid-JQA performs best.

Meanwhile, the Wait method is evaluated and the simulation results show that executing in available resource has better performance than waiting for proper resource. Finally, Dup Grid-JQA has minimum turnaround time and it is the best solution for matching in grid environment.

In future, we plan to implement our resource manager as a part of the Globus Toolkit and to do various experiments to measure the efficiency of the resource manager and job duplication. Also, we will investigate ways to select the best value of the threshold.

\section{References}

Bubendorfer, K. (2006). Fine grained resource reservation in open Grid economies, in: Proceedings of the 2nd IEEE International Conference on e-Science and Grid Computing, e-Science '06. 
Buyya, R.; Abramson, D.; Giddy, J. \& Stockinger, H. (1980). Economic models for resource management and scheduling in Grid computing, Concurrency and Computation: Practice and Experience 14, 1104_1113.

Chapin, S.; Karpovich, J. \& Grimshaw, A. (1999). The Legion Resource Management System, Proceedings oh the 5th Workshop on Job Scheduling Strategies for Parallel Processing, April 16, 1999, San Juan, Puerto Rico, USA, Springer Verlag Press, Germany.

Darby III, P.J. \& Tzeng, N.-F. (2010). Decentralized QoS-Aware Checkpointing Arrangement in Mobile Grid Computing. IEEE Trans. Mobile computing, vol. 9, no. 8,pp. 11731186, AUGUST. 2010.

Das, A. \& Grosu, D. (2005). Combinatorial Auction-Based Protocols for Resource Allocation in Grids, in: Proceedings of the 19th IEEE International Parallel and Distributed Processing Symposium, IPDPS'05.

Document for Web Services Agreement Specification, Open Grid Forum. http://www.ogf.org.

Foster, I.; Kesselman, C.; et al. (1999). A Distributed resource management architecture that supports advance reservation and co-allocation. In proceedings of the international workshop on Quality of Service, page 27-36

Foster, I. \& Kesselman, C. (2004). "The GRID: Blueprint for a New Computing Infrastructure", 2nd Edition.Morgan-Kaufmann, San Mateo, CA.

Goswami, K. \& Gupta, A. (2008). Resource selection in Grids using contract net, in: Proceedings of 16th Euromicro Conference on Parallel, Distributed and Network-Based Processing.

Han, Y. \& Youn, C.-H. (2009). A new grid resource management mechanism with resourceaware policy administrator for SLA-constrained applications, Future Generation Computer Systems 25,768_778.

Hewlett Packard Inc. espeak: The Universal Language of E-Services. http://www.espeak.net/.

Kakarontzas, G. \& Savvas, I.K. (2006). Agent-based resource discovery and selection for dynamic Grids, in: Proceedings of 15th IEEE International Workshops on Enabling Technologies: Infrastructure for Collaborative Enterprises, WETICE '06.

Khanli, L. M. \& Analoui, M. (2006a). “Grid-JQA - a new architecture for QoS-guaranteed grid computing system", Proc. of the 14th Euromicro Conference on Parallel, Distributed and Network-based processing, France, PDP2006, Feb 15-17.

Khanli, L. M. \& Analoui, M. (2006b). “Grid-JQA - grid java based quality of service management by active database", Proc. of the 4th Australian Symposium on Grid Computing and e-Research, AusGrid06, Jan.

Khanli, L. M. \& Analoui, M. (2008). An approach to Grid resource selection and fault management based on ECA rules, Future Generation Computer Systems 24 (4).

Kolano, P.Z. (2004). Surfer: An extensible pull-based framework for resource selection and ranking, in: Proceedings of the 4th IEEE International Symposium on Cluster Computing and the Grid.

Kondo, D. (2005). “Scheduling task parallel applications for rapid turnaround on desktop grids", Ph.D. thesis, university of California, San Diego 
Leal, K.; Huedo, E. \& Llorente, I.M. (2009). A decentralized model for scheduling independent tasks in federated Grid, Future Generation Computer Systems 25 (8) 840_852.

Litzkow, M.J.; Livny, M. \& Mutka, M.W. (1988). Condor: A Hunter of Idle Workstations, in: Proceedings of the 8th International Conference on Distributed Computing Systems.

Liu, C.; Yang, L.; Foster, I. \& Angulo, D. (2002). Design and Evaluation of a Resource Selection Framework for Grid Applications, in: Proceedings of the 11th IEEE International Symposium on High Performance Distributed Computing.

Liu, C. \& Foster, I. (2004). "A constraint language approach to matchmaking". In International workshop on Research Issues on Data Engineering (RIDE 2004).

Ludwig, S.A. \& Reyhani, S.M.S. (2006). Semantic approach to service discovery in a Grid environment, Future Generation Computer Systems 4 (1)1_13.

Neary, M.; Phipps, A.; Richman, S. \& Cappello, P. (2000). Javelin 2.0: Java-Based Parallel Computing on the Internet, Proceedings of European Parallel Computing Conference (Euro-Par 2000), Germany.

Raman, R.; Livny, M. \& Solomon, M. (1998). Matchmaking: Distributed resource management for high throughput computing, in: Proceedings of the 7th IEEE International Symposium on High Performance.

Raman, R.; Livny, M. \& Solomon, M. (2003). Policy driven heterogeneous resource coallocation with gangmatching, in: Proceedinsg of 12th IEEE International Symposium on High Performance Distributed Computing. In HPDC-12.

Rodero, I.; Guim, F.; Corbalan, J.; Fong, L. \& Sadjadi, S. M. (2010). Grid broker selection strategies using aggregated resource information, Future Generation Computer Systems 26 (2010) 72_86.

Sameer Singh Chauhan \& R. C. Joshi, "A Heuristic for QoS Based Independent Task Scheduling in Grid Environment", 5th International Conference on Industrial and Information Systems (ICIIS'10), Jul 29 - Aug 01, 2010a, India pp. 102-106.

Sameer Singh Chauhan \& R. C. Joshi, “A Weighted Mean Time Min- Min Max-Min Selective Scheduling Strategy for Independent Tasks on Grid", In proceedings of 2nd IEEE Advance Computing Conference, pp 4-9, February, 2010b.

Selvi Somasundaram, T.; Amarnath, B.R.; Kumar, R.; Balakrishnan, P.; Rajendar, K.; Rajiv, R.; Kannan, G.; Rajesh Britto, G.; Mahendran, E \& Madusudhanan, B. (2010). CARE Resource Broker: A framework for scheduling and supporting virtual, Future Generation Computer Systems 26 (2010) 337_347

Smith, R.G. (1980). The contract net protocol: High level communication and control in a distributed problem solver, IEEE Transactions on Computers 29 (12).

Tangmunarunkit, H.; Decker, S. \& Kesselman, C. (2003). Ontology-based Resource Matching in the Grid, in: Proceedings of workshop on Semantics in Peer-to- Peer and Grid Computing, SemPGRID'03.

Uddi.com. UDDI: Technical White Paper.http://www.uddi.com/pubs/Iru UDDI Technical White Paper.pdf.

Wang, C.-M.; Chen, H.-M.; Hsu, C.-C. \& Lee J. (2010). Dynamic resource selection heuristics for a non-reserved bidding-based Grid environment, Future Generation Computer Systems 26, 183_197. 
Xen Scheduler Howto. 2005: http://xen.terrabox.com/index.php/Sched-HOWTO.

Xiao, L.; Zhu, Y.; Ni, L.M. \& Xu, Z. (2008). Incentive-based scheduling for market-like computational Grids, IEEE Transactions on Parallel and Distributed Systems 19 (7).

Yu, H.; Bai, X. \& Marinescu, D.C. (2005). Workflow management and resource discovery for an intelligent Grid, Future Generation Computer Systems 31 (7) 797_811. 


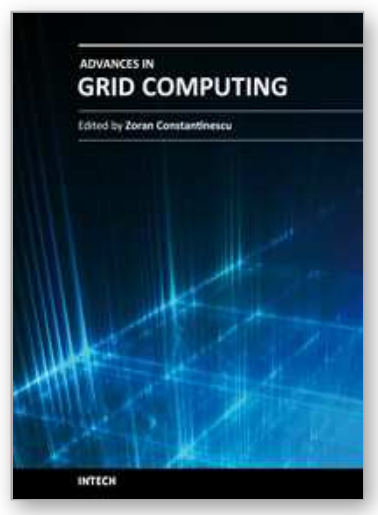

\author{
Advances in Grid Computing \\ Edited by Dr. Zoran Constantinescu
}

ISBN 978-953-307-301-9

Hard cover, 272 pages

Publisher InTech

Published online 28, February, 2011

Published in print edition February, 2011

This book approaches the grid computing with a perspective on the latest achievements in the field, providing an insight into the current research trends and advances, and presenting a large range of innovative research papers. The topics covered in this book include resource and data management, grid architectures and development, and grid-enabled applications. New ideas employing heuristic methods from swarm intelligence or genetic algorithm and quantum encryption are considered in order to explain two main aspects of grid computing: resource management and data management. The book addresses also some aspects of grid computing that regard architecture and development, and includes a diverse range of applications for grid computing, including possible human grid computing system, simulation of the fusion reaction, ubiquitous healthcare service provisioning and complex water systems.

\title{
How to reference
}

In order to correctly reference this scholarly work, feel free to copy and paste the following:

Leyli Mohammad Khanli and Saeed Kargar (2011). Grid-JQA: A QoS Guided Scheduling Algorithm for Grid Computing, Advances in Grid Computing, Dr. Zoran Constantinescu (Ed.), ISBN: 978-953-307-301-9, InTech, Available from: http://www.intechopen.com/books/advances-in-grid-computing/grid-jqa-a-qos-guidedscheduling-algorithm-for-grid-computing

\section{INTECH}

open science | open minds

\author{
InTech Europe \\ University Campus STeP Ri \\ Slavka Krautzeka 83/A \\ 51000 Rijeka, Croatia \\ Phone: +385 (51) 770447 \\ Fax: +385 (51) 686166 \\ www.intechopen.com
}

\author{
InTech China \\ Unit 405, Office Block, Hotel Equatorial Shanghai \\ No.65, Yan An Road (West), Shanghai, 200040, China \\ 中国上海市延安西路65号上海国际贵都大饭店办公楼 405 单元 \\ Phone: +86-21-62489820 \\ Fax: +86-21-62489821
}


(C) 2011 The Author(s). Licensee IntechOpen. This chapter is distributed under the terms of the Creative Commons Attribution-NonCommercialShareAlike-3.0 License, which permits use, distribution and reproduction for non-commercial purposes, provided the original is properly cited and derivative works building on this content are distributed under the same license. 